\title{
Evaluation of spectral ratio measures from spontaneous MEG recordings in patients with Alzheimer's disease
}

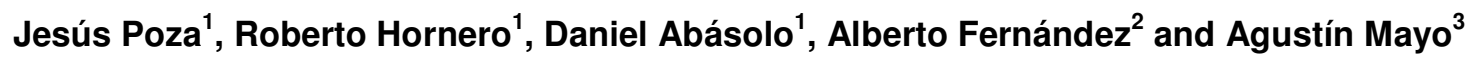

${ }^{1}$ Biomedical Engineering Group, E.T.S. Ingenieros de Telecomunicación, University of Valladolid, Spain

${ }^{2}$ Centro de Magnetoencefalografía Dr. Pérez-Modrego, Complutense University of Madrid, Spain

${ }^{3}$ Departamento de Estadística e Investigación Operativa, University of Valladolid, Spain

\section{Author's address:}

\section{Jesús Poza Crespo}

Biomedical Engineering Group, E.T.S. Ingenieros de Telecomunicación, University of Valladolid, Campus Miguel Delibes, Camino del Cementerio s/n, 47011 Valladolid, Spain

Phone: +34 983 423000, ext. 5569

Fax: +34 983423667

E-mail: jespoz@tel.uva.es

Url: www.gib.tel.uva.es 


\section{ABSTRACT}

Alzheimer's disease $(A D)$ is the most frequent form of dementia in western countries. The rise in life expectancy will likely increase its prevalence, since ageing is the greatest known risk factor. Although an early and accurate identification is critical, low diagnostic accuracy is currently reached. Hence, the aim of the present study was to analyse the spontaneous magnetoencephalographic (MEG) activity from 148 channels in $20 \mathrm{AD}$ patients and 21 healthy controls to extract discriminating spectral features. Relative power $(R P)$ was calculated in conventional frequency bands and several ratios were defined to emphasise the differences in its distribution. Both $R P$ values and spectral ratios were transformed with a principal component analysis to summarise information with minimal loss of variability. $\mathrm{AD}$ patients showed a significant increase of $R P(\delta)$ and $R P(\theta)$, along with a decrease of $R P(\beta)$ and $R P(\gamma)$. The most significant differences were reached by spectral ratios using the $\beta$ band. Specifically, we obtained $75.0 \%$ sensitivity, $90.5 \%$ specificity and $82.9 \%$ accuracy (linear discriminant analysis with a leave-one-out cross-validation procedure), together with a $p$-value lower than 0.001 (one-way analysis of variance with age as a covariate) using the $\left[R P(\alpha)+R P\left(\beta_{1}\right)+R P\left(\beta_{2}\right)+R P(\gamma]\right] /[R P(\delta)+R P(\theta)]$ ratio. The spectral ratios also showed a higher correlation with the severity of dementia than individual relative power measures. Our results suggest that the spectral ratios could be useful descriptors to help in the AD diagnosis, since they effectively summarise the slowing of the AD patients' MEG rhythms in individual indexes and correlate significantly with the severity of dementia.

Key Words: Alzheimer's disease; Magnetoencephalogram; Fourier transform; Spectral ratios; Principal component analysis 


\section{INTRODUCTION}

Alzheimer's disease $(A D)$ is a primary degenerative dementia of unknown aetiology. AD gradually destroys brain cells and leads to progressive decline in mental function, representing the most prevalent form of dementia in western countries [1]. Although several risk factors have been identified, the most important is ageing. This issue is especially significant in western countries due to the increase in life expectancy and, therefore, also in the prevalence of the disease [2].

Structural changes in $A D$ are related to the accumulation of amyloid plaques between nerve cells in the brain and with the appearance of neurofibrillary tangles inside nerve cells [3]. Both are considered as two AD hallmarks. However, it is not yet known whether plaques of tangles cause AD or are a by-product of some other process. They both are formed by clusters of proteins accumulated in greater quantities in two specific brain regions: the hippocampus and the cerebral cortex. The appearance of these two abnormal microscopic structures produces a neuronal damage or death, which is followed by a chemical imbalance. Both structural and chemical changes cause a progressive cell death and an overall shrinkage of brain tissue, which culminates in the progressive clinical symptoms of $A D[3]$.

Clinical diagnosis of $A D$ involves several kinds of evaluations: medical history study, mental status, memory, reasoning, vision-motor coordination, language skills evaluation, physical examination, brain scanning, laboratory tests, and psychiatric evaluation. Nevertheless, physicians only make a diagnosis of AD with sensitivity of around $81 \%$ and specificity of $70 \%$ [4]. A definite diagnosis of $A D$ can be only made by examining brain tissue after death. Despite the relatively low diagnostic accuracy, an early and accurate identification of AD should be attempted. Early detection is critical to optimise treatment, since current drugs are more effective if they are taken in the initial stages of the disease [5]. Furthermore, early identification will be even more critical when the new generation of drugs reach the clinic [6]. Finally, it is also noteworthy that an early diagnosis is important in the case of nonpharmacologic interventions, since it allows to develop a support system and review financial strategies [7].

In order to improve quality diagnosis, electrical brain activity has been widely analysed using electroencephalogram (EEG) recordings. Recently, special attention has also been given to magnetoencephalogram (MEG), which is a measure of the magnetic activity emitted by the brain. Both recordings are generated by synchronous oscillations of pyramidal neurons. However, EEG is 
sensitive to all primary currents, whereas MEG is insensitive to current flows oriented perpendicularly to the scalp. EEG and MEG detect slightly different features of the electromagnetic brain activity. Hence, they can be viewed as complementary techniques $[8,9]$. It should be pointed out that the cerebral magnetic fields are extremely weak. Hence, MEG recordings, at present, can be most conveniently detected by large arrays of superconducting quantum interference devices (SQUIDs). In order to maintain the SQUID sensors at the required low temperature, they are immersed in a cryogen housed in a thermally insulated container. Eventually, the MEG system is placed into a magnetically shielded room to attenuate the external noise. In this way, the technical requirements of MEG both increase the cost and reduce the flexibility of using this method in real-life clinical environments [9]. On the other hand, EEG power measurements are strongly influenced by a wide variety of non specified factors. Skull and other extracerebral tissues, technical and methodological issues (e.g. distance between sensors, electrode location, reference point or even conducting substance between skin and electrode) can affect the signal. An important advantage of MEG over EEG arises from the practical insensitivity of magnetic fields to tissue conductivities differences. In this sense, scalp EEG can be viewed as the result of a spatial filtering of the electrical cortical recordings by the volume conductor [10]. The effect of this process is to filter out high frequency components between the cortex and the scalp and, therefore, modify the spectral content of the cortical electric field [10]. This fact makes MEG recordings to be less distorted than EEG signals on the scalp [9]. In addition, magnetoencephalography obtains a reference-free recording as well as higher spatial resolution than conventional electroencephalography [8].

Some abnormalities in moderate and severe AD patients' EEG and MEG background activity have been observed [11-13]. AD patients show a decrease of coherence values at the $\alpha$ and $\beta$ bands in EEG [14,15], together with a slowing of spontaneous electrical brain activity. An increase of the $\delta$ (0.5-4 Hz) and $\theta(4-8 \mathrm{~Hz})$ power, along with a decrease of the $\alpha(8-13 \mathrm{~Hz})$ and $\beta(13-30 \mathrm{~Hz})$ power have been widely reported in EEG studies [16-19]. Results are in accordance with those obtained defining several power ratios to study EEG recordings [20-24]. Increase in slow activity has also been observed in the EEG spectral profile, where a spectrum shifted to the left [25] and the lack of a dominant activity in the $6.5-12 \mathrm{~Hz}$ frequency band [26] have been described. Similarly, several EEG studies considered some proper frequencies like mean frequency [19,20,25], individual alpha peak and transition frequency [27]. They found a decrease in characteristic frequencies associated to $A D$ patients' EEG compared with control subjects. It should be noticed that the EEG has been broadly 
investigated using spectral measures. However, only a few studies have analysed the MEG spectral patterns in AD. In this way, coherence analyses partially support the findings of previous EEG studies. Thus, a decrease of coherence values of the $\alpha$ band has been reported in AD patients' MEG recordings [28]. Nevertheless, a general decrease of coherence in all frequency bands has been also observed when analysing MEG signals [29]. Additionally, spontaneous MEG activity shows increased slow rhythms and reduced fast activity in AD patients in comparison with healthy subjects [29-31]. This fact has also been observed using some spectral parameters like mean frequency $[32,33]$ and individual alpha peak $[30,33,34]$.

The aim of the present work was to explore the ability of several spectral ratios to discriminate between $A D$ patients and elderly controls using MEG background recordings. The results were compared with those obtained with relative power in conventional frequency bands. Both relative power and spectral ratios were transformed using principal component analysis in order to summarise the large amount of information. While a few MEG studies have analysed the slowing of MEG rhythms using relative power measures, we propose to apply several ratio measures, as previous EEG studies have indicated. In this way, it should be possible to obtain an individual index which summarises all the spectral content simultaneously.

\section{MATERIALS AND METHODS}

\subsection{Selection of patients and controls}

Forty-one subjects participated in the study. Informed consent was obtained from all control subjects and all caregivers of the demented patients. The study was approved by the local ethics committee. All MEG recordings were performed in the "Centro de Magnetoencefalografía Dr. Pérez-Modrego" of the Complutense University of Madrid (Spain).

Twenty AD patients $(7$ men and 13 women, age $=73.1 \pm 8.7(56-83)$ years, mean \pm standard deviation SD (range)) were included in the study. All of them were recruited from the "Asociación de Familiares de Enfermos de Alzheimer" (AFAL). The diagnosis was made on the basis of exhaustive medical, physical, neurological, psychiatric and neuropsychological examinations. All patients fulfilled criteria for probable $A D$ according to the clinical guidelines of the National Institute of Communicative Disorders and Stroke - AD and Related Disorders Association (NINCDS-ADRDA) [35]. In addition, 
brain scans were performed to exclude other causes of dementia. Mini-Mental State Examination (MMSE) [36] and Functional Assessment Staging (FAST) [37] were used to assess the severity of the disease. The mean MMSE and FAST scores for the patients were $17.85 \pm 3.91$ and $4.00 \pm 0.32$ (Mean $\pm S D$ ), respectively. $A D$ patients were free from any other significant medical, neurological and psychiatric diseases. They were not taking any medication that could affect the recordings.

Twenty-one volunteers ( 9 men and 12 women, age $=70.3 \pm 7.1(56-84)$ years, mean \pm SD (range)) accepted to participate in the study as control group. They were cognitively normal elderly controls with no history of neurological or psychiatric disorders. Their mean MMSE and FAST scores were $29.10 \pm 1.00$ and $1.71 \pm 0.46$, respectively. No significant differences were detected in the mean age of both groups ( $p>0.05$, Student's t-test).

\subsection{MEG recording}

MEG signals were recorded using a 148-channel whole-head magnetometer (MAGNES 2500 WH, 4D Neuroimaging) confined in a magnetically shielded room in the "Centro de Magnetoencefalografía Dr. Pérez Modrego" (Spain). Recordings were obtained with subjects in a relaxed state, awake and with eyes closed in order to obtain as many artefact-free MEG data as possible. Vigilance was continuously monitored in order to avoid drowsiness. Five minutes of spontaneous MEG activity were acquired at a sampling rate of $678.17 \mathrm{~Hz}$. A $0.1-200 \mathrm{~Hz}$ hardware bandpass filter and a $50 \mathrm{~Hz}$ notch filter were also used. Subsequently, each signal recording was downsampled by a factor of four to reduce the data length.

Only artefact-free time segments of 1696 samples (10 s) were accepted to be used for further analysis. Off-line artefact rejection was performed by an experienced technician who was blind to the patients' diagnosis. In this way, an average number of $26 \pm 6$ artefact-free epochs (Mean \pm SD) for each subject and each channel were selected for subsequent calculations. It is noteworthy that $A D$ patients usually exhibited a higher degree of restlessness and agitation than healthy subjects. Therefore, a higher number of epochs had to be excluded in the AD patients' group than in the controls' one. Finally, prior to spectral analysis, each artefact-free segment of 1696 samples was digitally bandpass-filtered with cut-off frequencies at 0.4 and $70 \mathrm{~Hz}$. The selected frequency range enables to keep the relevant spectral content and to minimise the presence of electrooculographic and electromyographic artefacts. 


\subsection{Spectral analysis and ratio definition}

MEG segments were analysed using the Fourier transform (FT) in order to characterise their spectral properties. Power spectral density (PSD) was estimated from the FT of the autocorrelation function. PSD was averaged for each channel and subject to compute the mean PSD per channel and subject. Then, relative power $(R P)$ was calculated in the classical frequency bands: $\delta(1-4 \mathrm{~Hz}), \theta(4-8 \mathrm{~Hz}), \alpha(8-$ $13 \mathrm{~Hz}), \beta_{1}(13-19 \mathrm{~Hz}), \beta_{2}(19-30 \mathrm{~Hz})$ and $\gamma(30-64 \mathrm{~Hz})$.

Previous EEG studies have exploited the local differences in the relative power distribution defining a number of ratios [20-24]. Briefly, a balance between the power at high and at low frequencies was computed to summarise the slowing of the AD patients' EEG spectrum in a single index. To emphasise the local differences of the MEG power spectrum, we combined the relative power of conventional frequency bands, as it is shown in Equations (1), (2), (3) and (4). It should be noticed that $R P(\delta), R P(\theta), R P(\alpha), R P\left(\beta_{1}\right), R P\left(\beta_{2}\right)$ and $R P(\gamma)$ denote the relative power of each frequency band.

$$
\begin{gathered}
r_{1}=\frac{R P(\alpha)}{R P(\theta)} \\
r_{2}=\frac{R P(\alpha)+R P\left(\beta_{1}\right)+R P\left(\beta_{2}\right)+R P(\gamma)}{R P(\delta)+R P(\theta)} \\
r_{3}=\frac{R P\left(\beta_{1}\right)+R P\left(\beta_{2}\right)}{R P(\delta)} \\
r_{4}=\frac{R P\left(\beta_{2}\right)}{R P(\delta)} .
\end{gathered}
$$

Typically, the EEG slowing is more important with the severity of the disease [20,21]. Therefore, the performance of each spectral ratio will be established both by the frequency bands employed in their definition and by the severity of the demented patients. $r_{1}$ is a widely used ratio in previous EEG studies [20-22], which employs $\alpha$ and $\theta$ bands. Thus, $r_{1}$ is able to detect changes in early stages of the disease, when a slightly slowing in the EEG spectrum appears [20,21]. $r_{2}$ was used by Soininen et al. [20] and Bennys et al. [23] to obtain an index that summarised the EEG slowing globally. It should be noticed that in the aforementioned EEG studies the $\gamma$ band did not appear. Therefore, its contribution was lost. To take into account the $\gamma$ band, we extended the definition of $r_{2}$ to the whole power spectrum. Finally, some EEG works used the $R P(\alpha) / R P(\delta)$ ratio to detect changes in advanced stages of $\mathrm{AD}[20,22]$. In this study, we replaced $\alpha$ band by $\beta$ band, as it is shown in $r_{3}$ and 
$r_{4}$. This result is supported by a previous work, where the most significant changes in spontaneous MEG activity between $\mathrm{AD}$ patients and controls were observed in $\operatorname{RP}\left(\beta_{2}\right)$ [31]. Additionally, we did not only define a ratio to summarise the information of the whole $\beta$ band $\left(r_{3}\right)$, but also a more specific measure which quantifies the contribution of the $\beta_{2}$ band $\left(r_{4}\right)$.

In order to summarise information and detect correlation among the previous variables, principal component analysis (PCA) can be used. Briefly, PCA transforms a set of correlated parameters into a set of uncorrelated variables which are sorted by reducing variability. These new uncorrelated variables, derived from linear combinations of the original parameters, are called components. The coefficients of a linear combination are the components of an eigenvector of the correlation or covariance matrix. Thus, the first principal component is the combination of variables that explains the greatest amount of variability and so on. Finally, it is necessary to select an appropriate number of principal components. For this purpose, several methods have been proposed, like the broken stick model, Velicer's partial correlation procedure, cross-validation, bootstrapping techniques, Bartlett's test for equality of eigenvalues, Kaiser's criterion, Cattell's scree test and cumulative percentage of explained variance, among others [38]. However, it should be noticed that some authors point out that there is no ideal solution to the problem of dimensionality in a PCA [39] or that selection rules offer little advantage over simple schemes in most circumstances [38]. In this sense, previous EEG and MEG studies only retained the first principal component $\left(P C_{1}\right)$, since the explained variance for the calculated parameters was higher than $72 \%[32,33,40,41]$. In the present work, we applied a similar PCA scheme to summarise the variability of each parameter. But, in addition, the mean values for all channels were also computed to obtain a single measure per parameter and subject. Given the fact that the mean values showed a strong correlation with $P C_{1}$, we only selected the average measures for further analyses. In this way, a straightforward interpretation of the results is achieved, at the same time that more than $72 \%$ of variability is retained as it is shown in the results section.

\subsection{Statistical analysis}

Initially, a descriptive analysis was performed to explore the distribution of the parameters. Both the Kolmogorov-Smirnov and the Shapiro-Wilk tests were used to verify normality of distributions, whereas homoscedasticity was assessed with Levene's test. After the exploratory analysis, the values did not meet parametric test assumptions, since we observed asymmetry in the relative power and spectral 
ratio values. Therefore, a logarithmic transformation was applied to minimised problems with skewness and kurtosis [42], obtaining normally distributed variables. Then, a one-way analysis of variance (ANOVA) with age as a covariate was used to evaluate statistical significance $(\alpha=0.05)$. In addition, we used the Pearson correlation coefficient to study the relation between the parameters and both the MMSE and FAST scores.

Finally, the performance of the parameters to discriminate between groups was also evaluated by means of a linear discriminant analysis (LDA) with a leave-one-out cross-validation procedure. Classification results were summarised in terms of sensitivity (i.e. percentage of patients with a correct AD diagnosis), specificity (i.e. proportion of healthy subjects properly recognised) and accuracy (i.e. total fraction of subjects well classified).

\section{RESULTS}

We calculated the PSD of the $10 \mathrm{~s}$ segments in the 148 channels. Results were averaged for each channel and subject. Fig. 1 depicts the averaged normalised PSD (PSDn) for controls and AD patients. Mean relative power was then estimated for each classical frequency band. Finally, relative power values and spectral ratios were log-transformed and both a PCA and a grand-average over all channels were computed. Table 1 shows the percentage of variance explained by $P C_{1}$, together with the coefficient of determination between $P C_{1}$ and the mean of the parameters over all sensors. As it can be seen, the explained variance by the first principal components varied from $72.22 \%$ for the relative power value in the $\alpha$ band, to $88.01 \%$ in the $\beta_{2}$ band. Moreover, all spectral ratios were able to retain more than $83 \%$ of variance with just $P C_{1}$. These findings are in agreement with those obtained in previous MEG studies, which only analysed $P C_{1}$ due to the redundancy of the data $[32,33,41]$. Further inspection of Table 1 suggests that there exists also a strong correlation between each $P C_{1}$ and the corresponding mean for all channels ( $p<0.00001$ in all comparisons). In this way, to simplify the method, at the same time that a great amount of variability is retained, we analysed the mean over all log-transformed 148 parameters, instead of $P C_{1}$.

Insert Figure 1 about here

Insert Table 1 about here 
Table 2 summarises the correlations between each mean parameter and the age, the MMSE and FAST scores. It is noteworthy that in every case the obtained values were similar to those observed using Spearman correlation coefficient. Given the fact that the age is significantly correlated with several measures, its effect was eliminated from the statistical analysis by introducing the factor as a covariate. It should also be noticed that no significant relation with gender was found. Regarding to the MMSE and FAST scores, the most significant correlations were achieved by $r_{2}, r_{3}$ and $r_{4}$. According to their definitions, they were used to summarise the slowing of the power spectrum globally $\left(r_{2}\right)$ and to detect changes in advances stages of $\mathrm{AD}\left(r_{3}\right.$ and $\left.r_{4}\right)$. In this sense, an enhanced activity in lower frequency bands has been previously observed along with the severity of $A D[12,43,44]$, which is in agreement with the high correlation in the relative power of $\delta$ band.

\section{Insert Table 2 about here}

Fig. 2 depicts the notched boxplots with the relative power averaged for each group and for all channels. This kind of graph provides an excellent visual summary of many important aspects of a data distribution. The ordered values of the data are divided into four 'equal' parts, using a box with three horizontal lines. The sample median is displayed as a line approximately in the middle of the box, whereas the 25th and 75th percentiles of the sample are the lower and upper parts of the box, respectively. The notches included in the boxplot show a confidence interval of the median. They provide graphical information about the differences in the distributions by observing if the notches of the boxplots do not overlap. Finally, the 'whiskers' are two lines displayed from each end of the box to the farthest observation outside the box. They can be extended to a maximum of 1.5 times the interquartile range, whereas any values beyond these whiskers are defined as data outliers and marked by the symbol ' + '. $\delta$ and $\theta$ bands in AD patients show higher values than in control subjects ( $p$ $<0.01)$, while elderly controls have higher values with $\beta_{1}, \beta_{2}$ and $\gamma$ bands $(p<0.05) . \alpha$ band in control subjects shows higher relative power in comparison with AD patients. However, differences are not significant $(p>0.05)$.

Previous relative power values were combined in order to obtain the spectral ratios. As it can be noticed in Fig. 3, spectral ratios are higher in controls' MEGs than in AD patients' MEGs $(p<0.01)$. This result supports the increase in slow activities (i.e. $\delta$ and $\theta$ bands) and the decrease in fast 
rhythms (i.e. $\beta$ and $\gamma$ bands) observed for AD patients in Figs. 1 and 2. Moreover, differences are accentuated, given that the $p$-values are lower than those obtained using separate frequency bands.

Insert Figs. 2 and 3 about here

Sensitivity, specificity, accuracy and $p$-values for power values and spectral ratios are summarised in Table 3. In general, $p$-values for spectral ratios were lower than for relative power values, whereas accuracy was higher for spectral ratios than for individual power measures. Special attention should be paid to relative power value in $\beta_{1}$ and $\beta_{2}$ bands, which showed classification parameters similar to spectral ratios. It should be noticed that the best results were obtained with spectral measures that used the $\beta$ band. Thus, in both $\beta_{1}$ and $\beta_{2}$ bands the accuracy reached the $80.5 \%$ (70.0\%, sensitivity; $90.5 \%$, specificity). With regard to spectral ratios, the accuracy was $82.9 \%$ (75.0\%, sensitivity; $90.5 \%$, specificity) and $80.5 \%$ (75.0\%, sensitivity; $85.7 \%$, specificity) with $r_{2}$ and $r_{3}$, respectively.

Insert Table 3 about here

\section{DISCUSSION}

In the present study, we explored the ability of several spectral ratios to discriminate between spontaneous MEG oscillations from 20 AD patients and 21 control subjects. We calculated the relative power in classical frequency bands. PCA was then applied to both individual power values and spectral ratios in order to reduce the dimensionality of the problem. Due to the high correlation between $P C_{1}$ and the corresponding mean over all channels, in the sake of simplicity, we only consider the latter for further analyses. Significant differences for each average parameter were obtained using an ANOVA analysis with age as a covariate. Results revealed an increase in relative power estimations of AD patients at $\delta$ and $\theta$ bands. Controls showed higher relative power at $\beta$ and $\gamma$ bands. To emphasise differences in relative power distribution, we combined its values using several ratios. By retaining only the average measures over all channels, more significant differences were obtained with spectral ratios than using separate frequency bands. 
Further inspection of relative power values revealed that the most significant differences $(p<$ $0.001)$ were achieved in $\beta$ and $\delta$ bands. Significant differences were also obtained in $\theta(p<0.01)$ and $\gamma$ bands $(p<0.05)$. Our results suggested that relative power in low frequency bands were increased in $A D$. These findings are in agreement with previous studies analysing spontaneous MEG oscillations, where an increase in slow rhythms (i.e. $\delta$ and $\theta$ bands) and a decrease in fast oscillations (i.e. $\alpha$ and $\beta$ band) were reported [29-32]. In a similar way, EEG studies confirmed the slowing of brain rhythms [17-20,24-26]. A possible hypothesis to explain the EEG slowing in AD is the cholinergic deficit, which involves a loss of neurotransmitter acetylcholine [11]. Osipova et al. [45] studied the changes induced in spontaneous MEG activity by the administration of scopolamine. This medicament is a muscarinic receptor antagonist that blocks the stimulation of post-synaptic receptors and yields similar EEG alterations to those observed in AD. They found that the cholinergic system modulated spontaneous cortical activity at the $\theta$ and $\alpha$ bands and functional coupling in the $\theta$ band. Consequently, the cholinergic deficit could also be partly responsible of MEG slowing in $A D$, although due to the heterogeneity of the disease may not be sufficient to explain distinct alterations of the spontaneous MEG oscillations. More recently, Osipova et al. [30] performed a source analysis of MEG signals in $A D$. Their findings suggested that MEG slowing in $A D$ might not be due to the slowing of existing sources, but it might be owing to an increase in activation of low frequency generators. This hypothesis is supported by the findings of Fernández et al. [46,47], which observed an enhanced value in dipole density at $\delta$ and $\theta$ bands, when they analysed spontaneous MEG oscillations in AD. This result could be related to the increase of slow rhythms in AD. Nevertheless, more exhaustive studies are needed to confirm these hypotheses.

Results improved when we combined individual relative power values. We obtained more significant differences $(p<0.0001)$ with $r_{2}, r_{3}$ and $r_{4}$ than with relative power in conventional frequency bands. The lowest $p$-value $(p=0.000011<0.0001)$ was obtained with $r_{2}$. Moreover, with $r_{2}$ optimum classification parameters of $75.0 \%$ sensitivity, $90.5 \%$ specificity and $82.9 \%$ accuracy were achieved. It should be noticed that both $r_{3}$ and $r_{4}$ used $\beta$ and $\delta$ bands in their definition. These results suggest a significant increase of $\delta$ power and a parallel decrease in $\beta$ oscillations, as it can be noticed in Fig. 2 . This issue is supported by a previous MEG study, which performed an exhaustive frequency analysis in $A D$ [31]. Hence, two well differentiated regions of interest between 2 to $4 \mathrm{~Hz}$ and 16 to $28 \mathrm{~Hz}$ were found when the spectrum of $A D$ patients and controls was compared [31]. The important role of $\beta$ and 
$\delta$ bands in AD characterisation has also been observed in several EEG works [20-24]. Table 4 summarises the results obtained by previous studies that analysed the EEG activity using spectral ratios to explore the dynamics associated with $A D$.

Insert Table 4 about here

Special attention to $\beta$ band has also been paid by Bennys et al. [23]. In an analogous EEG study using spectral ratios, they found significant differences between $A D$ patients and control subjects. They employed two power ratios similar to $r_{1}$ and $r_{2}$. It is noteworthy that, unlike our study, they discarded the use of $\delta$ band due to electrooculographic activity and suggested the use of $\theta$ band in the spectral ratio definition. Nevertheless, our results suggest that $\delta$ band should be taken into consideration in the characterisation of AD patients' electromagnetic brain activity, as it has been showed with $r_{2}, r_{3}$ and $r_{4}$. In this sense, Leuchter et al. [21] observed that the highest classification rates were achieved using parameters based on low frequency bands (i.e. $\delta$ and $\theta$ bands), when they studied regional differences in the EEG between demented patients and controls. Furthermore, their findings suggested that the parameters yielded complementary information and could be then sensitive to different pathologic processes in dementia [21]. Finally, the usefulness of $\delta$ band has also been proved by Jelic et al. [22]. They showed a strong correlation between cerebrospinal fluid (CSF) tau levels and two spectral ratios, when the EEG activity from $A D$ patients was analysed.

On the other hand, Soininen et al. [20] did not obtain significant differences in $\beta$ power between groups, but a significant slowing of the power spectrum was reported. Results improved when several ratios were defined. Soininen et al. [20] concluded that the EEG slowing was different at the time of $A D$ diagnosis and 1 year later, due to the heterogeneity of the disease for each patient. In this sense, Matousek et al. [24] found that the $R P(\theta) / R P(\alpha)$ ratio was more correlated to the degree of dementia than individual power measures. Moreover, the EEG slowing was highly dependent on the type of regional syndrome affecting each patient. In a similar sense, our findings also suggest that the spectral ratios show a stronger correlation with the severity of dementia than individual relative power measures. However, in the present study the patients are slightly more demented than in the work carried out by Matousek et al. [24]. Therefore, we observed that the highest correlation was found with 
$r_{2}$. In addition, it is noteworthy that $r_{3}$ and $r_{4}$ exhibited correlation coefficients close to those obtained with $r_{2}$, which indicated an advanced degree of dementia.

EEG and MEG slowing have not only been observed in $A D$, but also in other forms of dementia as mild cognitive impairment [18,19,32], vascular dementia [25,27], dementia associated with Parkinson's disease [26,48], Lewy body dementia [26,28,49], major depression [50,51], Huntington's chorea and progressive supranuclear palsy $[16,26]$. Further investigation should be attempted in order to study differences in slowing for every disorder. In a similar way, it might be possible that the increase in relative power of slow frequencies could be drug-related. Nevertheless, in our study, none of patients were receiving any medication which could affect spontaneous MEG activity.

Another important limitation of our study is related to the small sample size. Thus, considerations about type I (incorrectly deciding to reject a null hypothesis) and type II (incorrectly deciding not to reject a null hypothesis) errors should be made. The reduced number of subjects involves an increase of beta (probability of making a type II error) and of the probability of a false negative. To overcome the problem and to ensure generalisation, results should be extended on a larger population.

Finally, it is noteworthy that, in the sake of simplicity, the present approach involves a loss of spatial information, since the parameters were averaged over the 148 channels. A partial solution could be based on computing the average of each index for several brain regions, taking into account that the MEG activity recorded by a sensor could not reflect only the brain rhythms under the channel, but it might also measure the activity from other areas. In this sense, further works could be carried out to explore the spatial patterns for each parameter.

\section{CONCLUSIONS}

Life conditions in western countries have notably improved during the last decades. This issue involves an increase in life expectancy and, therefore, in the prevalence of neurodegenerative diseases associated with ageing, such as $A D$. To the previous issue, the difficulties faced in $A D$ identification, like the low diagnostic accuracy, should be added. Nevertheless, an early and accurate identification should be attempted both to optimise the treatment and to develop strategies for coping with the disease. In this sense, it is worth noting that $A D$ is a cortical degenerative dementia affecting the electromagnetic brain activity. Hence, both the EEG and MEG are expected to reflect functional 
and structural deficits of the brain damaged by the disease. While the spectral features of the EEG have been extensively analysed, only a few MEG studies have explored the ability of spectral measures as AD descriptors [29-33]. In this sense, to the best of our knowledge, this study is the first approximation to demonstrate the ability of spectral ratios to extract discriminating features from $A D$ patients' MEG background activity in comparison to elderly controls. Our results support the notion that $A D$ involves a slowing of electromagnetic brain activity when compared with elderly subjects and point out the importance of $\beta$ band in the understanding of the disease. Additionally, spectral ratios correlate more significantly with the severity of the dementia than individual power measures, suggesting a potential usefulness of these indexes. Further works will be carried out to explore the spatial patterns for each parameter using a larger data set, including patients suffering from other neurodegenerative diseases.

In summary, our findings suggest that the spectral ratios can be useful descriptors of the spontaneous MEG rhythms in AD. Moreover, the significant correlation between these measures and the severity of dementia can provide further insights on brain dynamics in $A D$.

Acknowledgements This work has been partially supported by the grant project VA108A06 from "Consejería de Educación de Castilla y León" and by the "Ministerio de Educación y Ciencia" grant SEJ2004-06969. The authors would like to thank the "Asociación de Enfermos de Alzheimer" (AFAL) for supplying the patients who have participated in this study and the Reviewers for their critical feedback on the original manuscript. 


\section{REFERENCES}

[1] T.D. Bird, Alzheimer's disease and other primary dementias, in Harrison's principles of internal medicine, eds. E. Braunwald, A.S. Fauci, D.L. Kasper, S.L. Hauser, D.L. Longo and J.L. Jameson, pp. 2391-2399 (The McGraw-Hill Companies Inc., New York NY, 2001).

[2] K. Blennow, M.J. de Leon and H. Zetterberg, Alzheimer's disease, Lancet 368 (2006) 387-403.

[3] R. Katzman, Current research on Alzheimer's disease in a historical perspective, in Diagnosis, Treatment and Care, eds. Z.S. Khachaturian and T.S. Radebaugh, pp. 15-29 (CRC Press, Boca Raton FL, 1996).

[4] D.S. Knopman, S.T. DeKosky, J.L. Cummings, H. Chui, J. Corey-Bloom, N. Relkin, G.W. Small, B. Miller and J.C. Stevens, Practice parameter: Diagnosis of dementia (an evidence-based review). Report of the Quality Standards Subcommittee of the American Academy of Neurology, Neurology 56 (2001) 11431153.

[5] J.L. Cummings, Alzheimer's disease, N. Engl. J. Med. 351 (2004) 56-67.

[6] K. Blennow, CSF biomarkers for mild cognitive impairment, J. Intern. Med. 256 (2004) 224-234.

[7] P.M. Aupperle, Navigating patients and caregivers through the course of Alzheimer's disease, J. Clin. Psychiatry 67 (2006) 8-14.

[8] M. Hämäläinen, R. Hari, R.J. IImoniemi, J. Knuutila and O.V. Lounasmaa, Magnetoencephalograpy theory, instrumentation, and applications to noninvasive studies of the working human brain, Rev. Mod. Phys. 65 (1993) 413-497.

[9] R. Hari, Magnetoencephalography in clinical neurophysiological assessment of human cortical functions, in Electroencephalography: Basic Principles, Clinical Applications, and Related Fields, eds. E. Niedermeyer and F. Lopes da Silva, pp. 1165-1197 (Lippincontt Willians \& Wilkins, Philadelphia PA, 2005).

[10] P.L. Nunez, B.M. Wingeier and R.B. Silberstein, Spatial-Temporal structures of human alpha rhythms: Microcurrents sources, multiscale elements, and global binding of local networks, Hum. Brain Mapp. 13 (2001) 125-164.

[11] J. Jeong, EEG dynamics in patients with Alzheimer's disease, Clin. Neurophysiol. 115 (2004) 1490-1505.

[12] H. Adeli, G.-D- Samanwoy and N. Dadmehr, Alzheimer's disease: models of computation and analysis of EEGs, Clin. EEG Neurosci. 36 (2005) 131-140.

[13] C.J. Stam, T. Montez, B.F. Jones, S.A.R.B. Rombouts, Y. van der Made, Y.A.L. Pijnenburg and Ph. Scheltens, Disturbed fluctuations of resting state EEG synchronization in Alzheimer's disease, Clin. Neurophysiol. 116 (2005) 708-715.

[14] C. Besthorn, H. Förstl, C. Geiger-Kabisch, H. Sattel, T. Gasser and U. Schreiter-Gasser, EEG coherence in Alzheimer disease, Electroenceph. Clin. Neurophysiol. 90 (1994) 242-245. 
[15] G. Adler, S. Brassen and A. Jajcevic, EEG coherence in Alzheimer's dementia, J. Neural Transm. 110 (2003) 1051-1058.

[16] K. Maurer and T. Dierks, Functional imaging procedures in dementias: mapping of EEG and evoked potentials, Acta Neurol. Scand. Suppl. 139 (1992) 40-46.

[17] U. Schreiter-Gasser, T. Gasser and P. Ziegler, Quantitative EEG analysis in early onset Alzheimer's disease: a controlled study, Electroenceph. Clin. Neurophysiol. 86 (1993) 15-22.

[18] C. Huang, L. Wahlund, T. Dierks, P. Julin, B. Winblad and V. Jelic, Discrimination of Alzheimer's disease and mild cognitive impairment by equivalent EEG sources: a cross-sectional and longitudinal study, Clin. Neurophysiol. 111 (2000) 961-1967.

[19] V. Jelic, S.-E. Johansson, O. Almkvist, M. Shigeta, P. Julin, A. Nordberg, B. Winblad and L.-O. Wahlund, Quantitative electroencephalography in mild cognitive impairment: longitudinal changes and possible prediction of Alzheimer's disease, Neurobiol. Aging 21 (2000) 533-540.

[20] H. Soininen, J. Partanen, V. Laulumaa, E.-L. Helkala, M. Laakso and P.J. Riekkinen, Longitudinal EEG spectral analysis in early stage of Alzheimer's disease, Electroenceph. Clin. Neurophysiol. 72 (1989) 290297.

[21] A.F. Leuchter, I.A. Cook, T.F. Newton, J. Dunkin, D.O. Walter, S. Rosenberg-Thompson, P.A. Lachenbruch and $\mathrm{H}$. Weiner, Regional differences in brain electrical activity in dementia: use of spectral power and spectral ratio measures, Electroenceph. Clin. Neurophysiol. 87 (1993) 385-393.

[22] V. Jelic, M. Blomberg, T. Dierks, H. Basun, M. Shigeta, P. Julin, M. Jensen, L. Lannfelt, B. Winblad and L.O. Wahlund, EEG slowing and cerebrospinal fluid tau levels in patients with cognitive decline, Neuroreport 9 (1998) 157-160.

[23] K. Bennys, G. Rondouin, C. Vergnes and J. Touchon, Diagnostic value of quantitative EEG in Alzheimer's disease, Neurophysiol. Clin. 31 (2001) 153-160.

[24] M. Matousek, M. Brunovsky, A. Edman and A. Wallin, EEG abnormalities in dementia reflect the parietal lobe syndrome, Clin. Neurophysiol. 112 (2001) 1001-1005.

[25] M. Signorino, E. Pucci, N. Belardinelli, G. Nolle and F. Angeleri, EEG spectral analysis in vascular and Alzheimer dementia, Electroenceph. Clin. Neurophysiol. 94 (1995) 313-325.

[26] E. Pucci, G. Cacchiò, R. Angeloni, N. Belardinelli, G. Nolfe, M. Signorino and F. Angeleri, EEG spectral analysis in Alzheimer's disease and different degenerative dementias, Arch. Gerontol. Geriatr. 26 (1998) 283-297.

[27] D.V. Moretti, C. Babiloni, G. Binetti, E. Cassetta, G. Dal Forno, F. Ferreric, R. Ferri, B. Lanuzza, C. Miniussi, F. Nobili, G. Rodriguez, S. Salinari and P.M. Rossini, Individual analysis of EEG frequency and band power in mild Alzheimer's disease, Clin. Neurophysiol. 115 (2004) 299-308. 
[28] R. Franciotti, D. lacono, S. Della Penna, V. Pizzella, K. Torquati, M. Onofrj and G.L. Romani, Cortical rhythms reactivity in AD, LBD and normal subjects. A quantitative MEG study, Neurobiol. Aging 27 (2006) $1100-1109$.

[29] H.W. Berendse, J.P.A. Verbunt, Ph. Scheltens, B.W. van Dijk and E.J. Jonkman, Magnetoencephalographic analysis of cortical activity in Alzheimer's disease: a pilot study, Clin. Neurophysiol. 111 (2000) 604-612.

[30] D. Osipova, J. Ahveninen, O. Jensen, A. Ylikoski and E. Pekkonen, Altered generation of spontaneous oscillations in Alzheimer's disease, Neuroimage 27 (2005) 835-841.

[31] A. Fernández, R. Hornero, A. Mayo, J. Poza, F. Maestú and T. Ortiz, Quantitative magnetoencephalography of spontaneous brain activity in Alzheimer disease: an exhaustive frequency analysis, Alzheimer Dis. Assoc. Disord. 20 (2006) 153-159.

[32] A. Fernández, R. Hornero, A. Mayo, J. Poza, P. Gil-Gregorio and T. Ortiz, MEG spectral profile in Alzheimer's disease and mild cognitive impairment, Clin. Neurophysiol. 117 (2006) 306-314.

[33] J. Poza, R. Hornero, D. Abásolo, A. Fernández and M. García, Extraction of spectral based measures from MEG background oscillations in Alzheimer's disease, Med. Eng. Phys. 29 (2007), 1073-1083.

[34] D. Osipova, K. Rantanen, J. Ahveninen, R. Ylikoski, O. Happola, T. Strandberg and E. Pekkonen, Source estimation of spontaneous MEG oscillations in mild cognitive impairment, Neurosci. Lett. 405 (2006) 5761.

[35] G. McKhann, D. Drachman and M. Folstein, Clinical diagnosis of Alzheimer's disease: report of NINCDSADRDA work group under the auspices of department of health and human services task force on Alzheimer's disease, Neurology 34 (1984) 939-944.

[36] M.F. Folstein, S.E. Folstein and P.R. McHugh, Mini-mental state. A practical method for grading the cognitive state of patients for the clinician, J. Psychiatr. Res. 12 (1975) 189-198.

[37] B. Reisberg, Functional assessment staging (FAST), Psychopharmacol. Bull. 24 (1988) 653-659.

[38] I.T. Jolliffe, Principal Component Analysis (Springer-Verlag, New York NY, 1986).

[39] L. Ferré L, Selection of components in principal component analysis: A comparison of methods, Computat. Statist. Data Anal. 19 (1995) 669-682.

[40] T. Müller, T. Ball, R. Kristeva-Feige, T. Mergner and J. Timmer, Selecting relevant electrode positions for classification tasks based on the electro-encephalogram, Med. Biol. Eng. Comput. 38 (2000) 62-67.

[41] C. Gómez, R. Hornero, D. Abásolo, A. Fernández and M. López, Complexity analysis of the magnetoencephalogram background activity in Alzheimer's disease patients, Med. Eng. Phys. 28 (2006) 851-859. 
[42] V. Pollock, L. Schneider and S. Lyness, EEG amplitudes in healthy, late-middle-aged and elderly adults: normality of the distributions and correlations with age, Elecotroenceph. Clin. Neurophysiol. 75 (1990) 276-288.

[43] L.A. Coben, W. Danziger and M. Storandt, A longitudinal EEG study of mild senile dementia of Alzheimer type: changes at 1 year and at 2.5 years, Electroencephalogr. Clin. Neurophysiol. 61 (1985) 101-112.

[44] H. Soininen and P.J. Riekkinen, EEG in diagnostics and follow-up of Alzheimer's disease, Acta Neurol. Scand. (1992) 36-39.

[45] D. Osipova, J. Ahveninen, S. Kaakkola, I.P. Jääskeläinen, J. Huttunen and E. Pekkonen, Effects of scopolamine on MEG spectral power and coherence in elderly subjects, Clin. Neurophysiol. 114 (2003) 1902-1907.

[46] A. Fernández, F. Maestú, C. Amo, P. Gil, T. Fehr, C. Wienbruch, B. Rockstroh, T. Elbert and T. Ortiz, Focal temporoparietal slow activity in Alzheimer's disease revealed by magnetoencephalography, Biol. Psychiatry 52 (2002) 764-770.

[47] A. Fernández, A. Turrero, P. Zuluaga, P. Gil, F. Maestú, P. Campo and T. Ortiz, Magnetoencephalographic parietal delta dipole density in mild cognitive impairment: preliminary results of a method to estimate the risk of developing Alzheimer disease, Arch. Neurol. 63 (2006) 427-430.

[48] J.C. Pin, J.P. Neau, J. Paquereau, C. Rostykus, D. Guehl, I. Aubert, A. Marillaud and R. Gil, Comparative study by computerized EEG of dementia of the Alzheimer type and Parkinson's disease with dementia, Neurophysiol. Clin. 22 (1992) 301-312.

[49] T. Kai, Y. Asai, K. Sakuma, T. Koeda and K. Nakashima, Quantitative electroencephalogram analysis in dementia with Lewy bodies and Alzheimer's disease, J. Neurol. Sci. 237 (2005) 89-95.

[50] R.P. Brenner, R.F. Ulrich, D.G. Spiker, R.J. Sclabassi, C.F. Reynolds III, R.S. Marin and F. Boller, Computerized EEG spectral analysis in elderly normal, demented and depressed subjects, Electroenceph. Clin. Neurophysiol. 64 (1986) 483-492.

[51] P.N. Prinz and M.V. Vitiello, Dominant occipital (alpha) rhythm frequency in early stage Alzheimer's disease and depression, Electroenceph. Clin. Neurophysiol. 73 (1989) 427-432. 


\section{TABLE CAPTIONS}

Table 1. Percentage of variance explained by the first principal component (\% Var. $\left.P C_{1}\right)$ for all frequency bands and spectral ratios, together with the coefficient of determination $\left(R^{2}\right)$ between each $P C_{1}$ and the corresponding mean values over all channels

Table 2. Pearson correlation coefficients $(r)$ between the mean values over all channels and the age, the MMSE and the FAST scores

Table 3. Classification results of linear discriminant analysis with a leave-one-out cross-validation procedure, together with the corresponding $p$-values from ANOVA corrected for age. Both analyses were performed using the mean values over all channels for each parameter

Table 4. Summary of previous EEG studies, in alphabetical order, concerning the analysis of AD by means of spectral ratios 


\section{FIGURE LEGENDS}

Fig. 1. Normalised power spectral density (PSDn) functions averaged over all channels for healthy controls $(C)$ and $A D$ patients $(A D)$.

Fig. 2. Notched boxplots displaying the distribution of the mean relative power values averaged for all channels at each frequency band and the corresponding $p$-values: (a) $R P(\delta)$ (AD patients: $0.32 \pm 0.13$; Control subjects: $0.17 \pm 0.06$, mean $\pm \mathrm{SD}$ ); (b) $R P(\theta)$ (AD patients: $0.22 \pm 0.10$; Control subjects: 0.15 $\pm 0.05)$; (c) $R P(\alpha)$ (AD patients: $0.18 \pm 0.08$; Control subjects: $0.21 \pm 0.08$ ); (d) $R P\left(\beta_{1}\right)$ (AD patients: $0.11 \pm 0.05$; Control subjects: $0.18 \pm 0.04$ ); (e) $R P\left(\beta_{2}\right)$ (AD patients: $0.10 \pm 0.05$; Control subjects: 0.20 $\pm 0.08)$; (f) $R P(\gamma)$ (AD patients: $0.06 \pm 0.03$; Control subjects: $0.09 \pm 0.04)$.

Fig. 3. Notched boxplots displaying the distribution of the mean spectral ratios averaged for all channels and the corresponding $p$-values: (a) $r_{1}$ (AD patients: $1.05 \pm 0.78$; Control subjects: $1.62 \pm$ 0.78, mean \pm SD); (b) $r_{2}$ (AD patients: $1.20 \pm 0.98$; Control subjects: $2.65 \pm 1.04$ ); (c) $r_{3}$ (AD patients: $1.20 \pm 1.29$; Control subjects: $3.10 \pm 1.46$ ); (d) $r_{4}$ (AD patients: $0.56 \pm 0.55$; Control subjects: $1.59 \pm$ 0.84). 
Figure 1

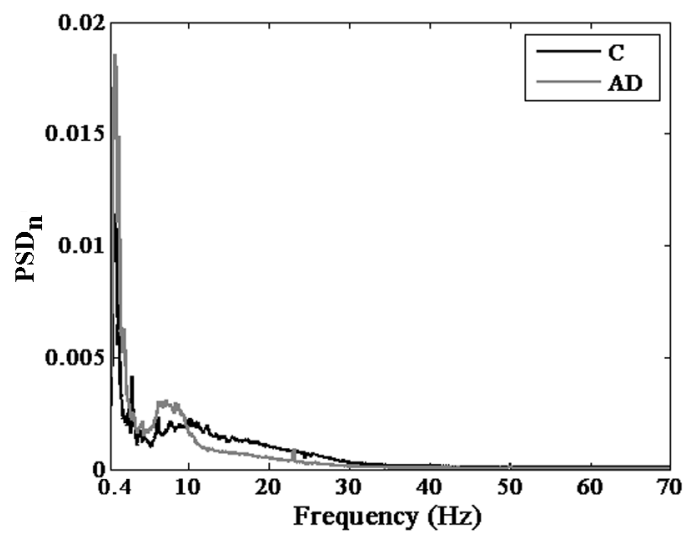


Figure 2

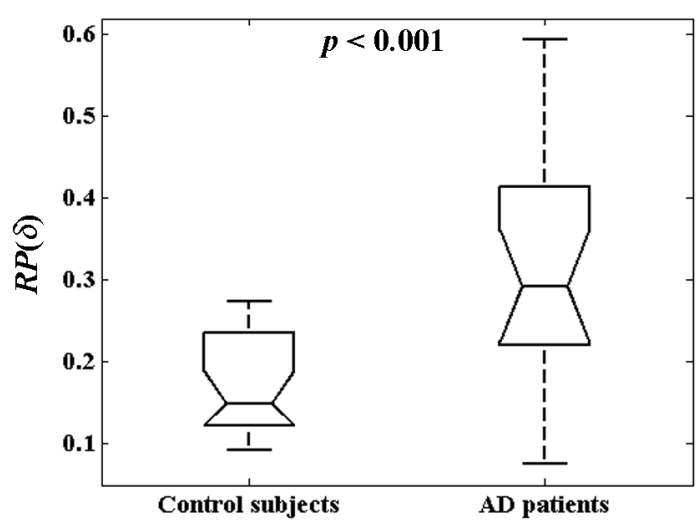

(a)

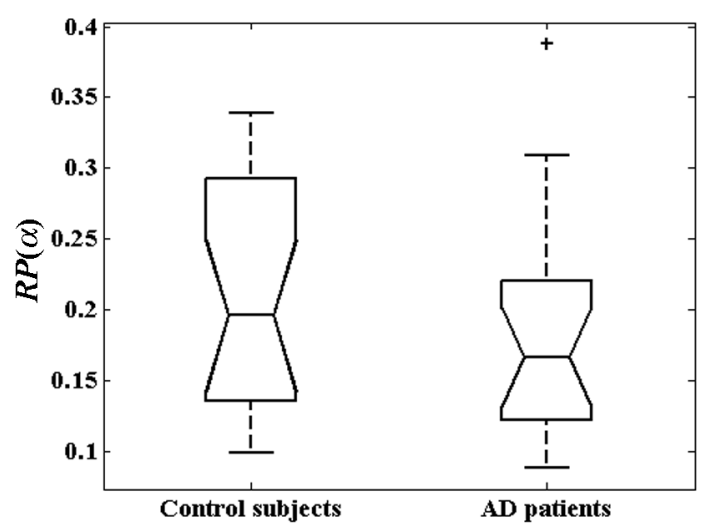

(c)

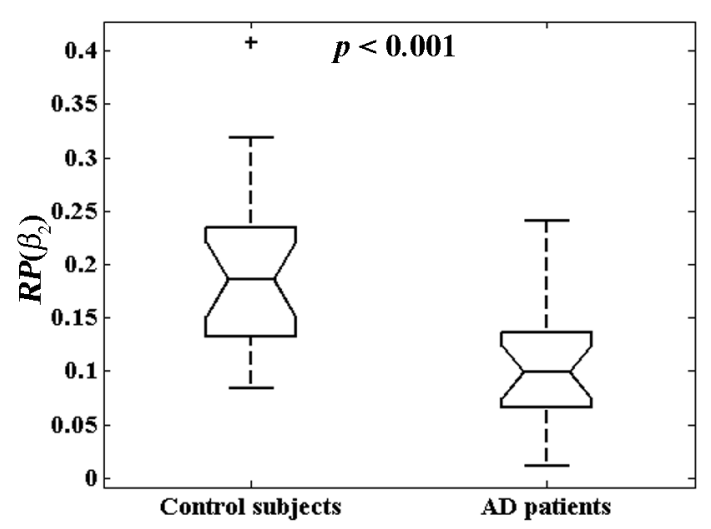

(e)

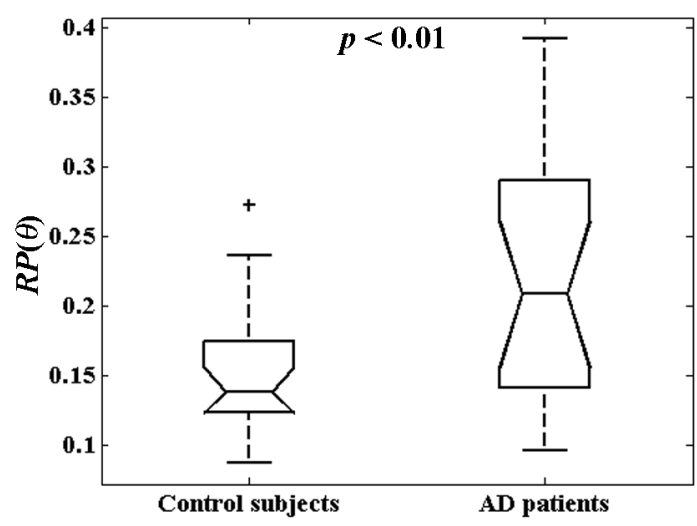

(b)

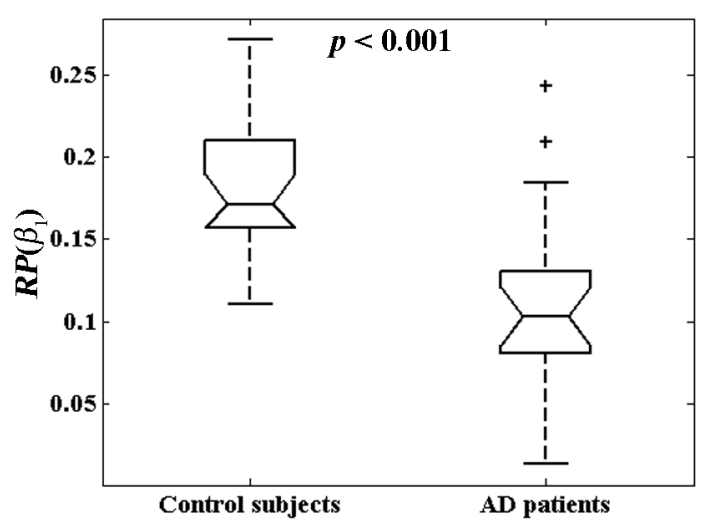

(d)

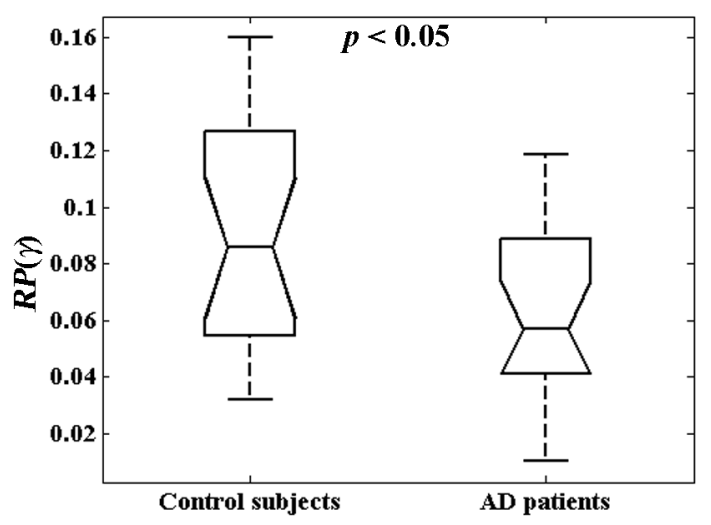

(f) 
Figure 3

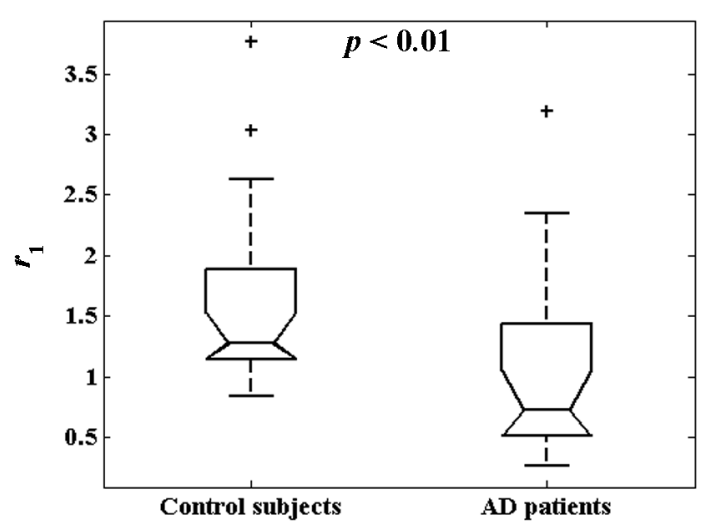

(a)

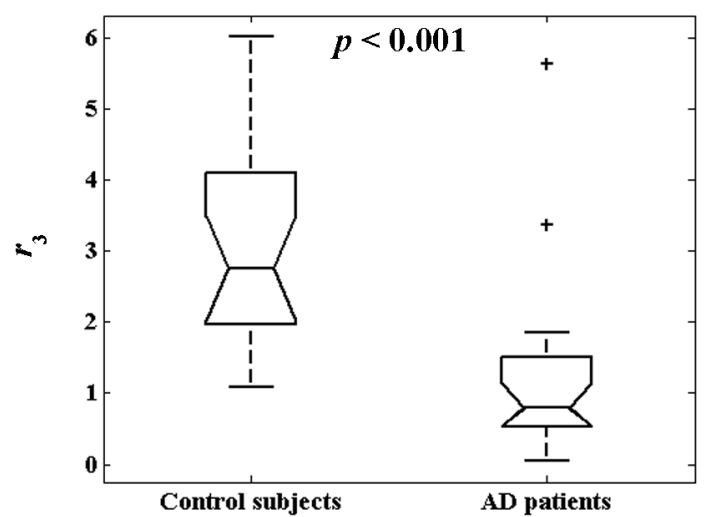

(c)

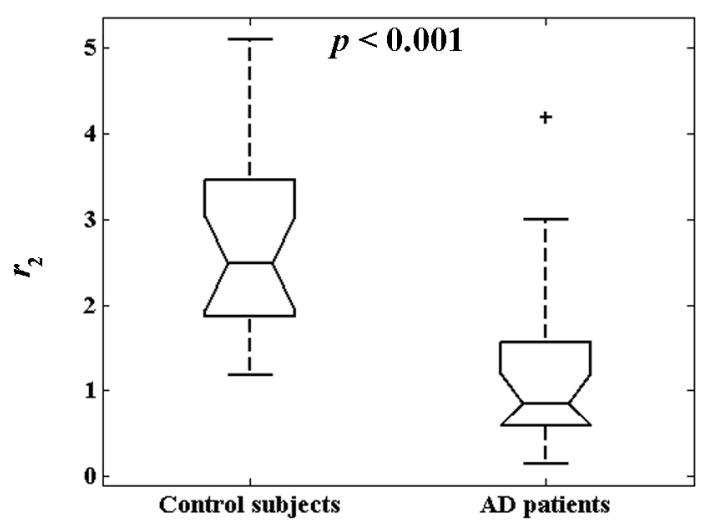

(b)

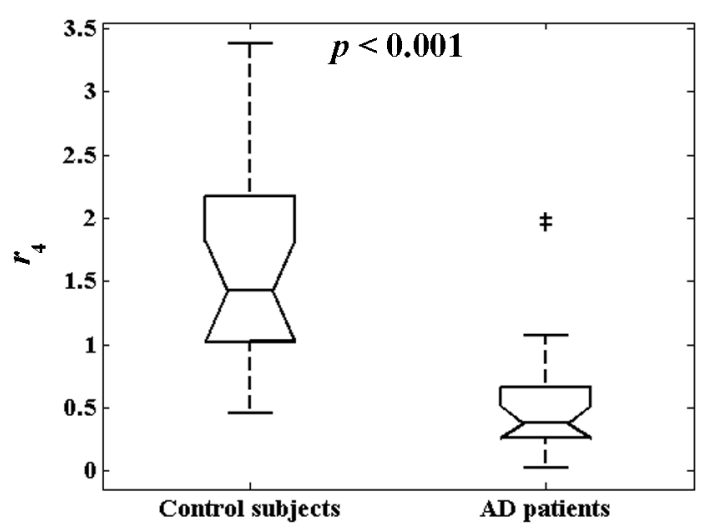

(d) 
Table 1

\begin{tabular}{|c|c|c|c|}
\hline \multicolumn{2}{|c|}{ Parameters } & \multirow{2}{*}{$\frac{\% \text { Var. } P C_{1}}{81.48}$} & \multirow{2}{*}{$\begin{array}{c}R^{2} \\
0.99964\end{array}$} \\
\hline \multirow{6}{*}{$\begin{array}{l}\text { Relative } \\
\text { power }\end{array}$} & $R P(\delta)$ & & \\
\hline & $R P(\theta)$ & 77.22 & 0.99763 \\
\hline & $R P(\alpha)$ & 72.22 & 0.99944 \\
\hline & $R P\left(\beta_{1}\right)$ & 83.13 & 0.99962 \\
\hline & $R P\left(\beta_{2}\right)$ & 88.01 & 0.99972 \\
\hline & $R P(\lambda)$ & 83.39 & 0.99858 \\
\hline \multirow{4}{*}{$\begin{array}{l}\text { Spectral } \\
\text { ratios }\end{array}$} & $r_{1}$ & 83.65 & 0.99914 \\
\hline & $r_{2}$ & 84.21 & 0.99989 \\
\hline & $r_{3}$ & 86.11 & 0.99995 \\
\hline & $r_{4}$ & 87.07 & 0.99992 \\
\hline
\end{tabular}


Table 2

\begin{tabular}{llcccccc}
\hline \multirow{2}{*}{ Parameters } & \multicolumn{2}{c}{ Age } & \multicolumn{2}{c}{ MMSE } & \multicolumn{2}{c}{ FAST } \\
\cline { 3 - 7 } & & $r$ & $p$-value & $r$ & $p$-value & $r$ & $p$-value \\
\hline \multirow{4}{*}{ Relative } & $R P(\delta)$ & 0.29628 & 0.059978 & -0.53130 & 0.000351 & 0.57052 & 0.000098 \\
power & $R P(\theta)$ & 0.48492 & 0.001313 & -0.35818 & 0.021483 & 0.41321 & 0.007249 \\
& $R P\left(\beta_{1}\right)$ & 0.11680 & 0.467063 & 0.28533 & 0.070564 & -0.19954 & 0.211000 \\
& $R P\left(\beta_{2}\right)$ & -0.29332 & 0.062707 & 0.41225 & 0.007399 & -0.50449 & 0.000770 \\
& $R P(\gamma)$ & -0.47505 & 0.001698 & 0.22553 & 0.156263 & -0.33805 & 0.030638 \\
\hline \multirow{3}{*}{ Spectral } & $r_{1}$ & -0.25696 & 0.104845 & 0.44015 & 0.003980 & -0.42045 & 0.006199 \\
ratios & $r_{2}$ & -0.42960 & 0.005062 & 0.54683 & 0.000216 & -0.60202 & 0.000031 \\
& $r_{3}$ & -0.38160 & 0.013823 & 0.51896 & 0.000508 & -0.58739 & 0.000054 \\
& $r_{4}$ & -0.43426 & 0.004556 & 0.51509 & 0.000569 & -0.58417 & 0.000061 \\
\hline
\end{tabular}




\section{Table 3}

\begin{tabular}{llllll}
\hline \multicolumn{2}{c}{ Parameters } & Sensitivity (\%) & Specificity (\%) & Accuracy (\%) & $p$-value \\
\hline \multirow{4}{*}{ Relative } & $R P(\delta)$ & $15 / 20(75.0)$ & $16 / 21(76.2)$ & $31 / 41(75.6)$ & 0.000101 \\
power & $R P(\theta)$ & $13 / 20(65.0)$ & $17 / 21(81.0)$ & $30 / 41(73.2)$ & 0.007813 \\
& $R P(\alpha)$ & $12 / 20(60.0)$ & $12 / 21(57.1)$ & $24 / 41(58.5)$ & 0.120047 \\
& $R P\left(\beta_{1}\right)$ & $14 / 20(70.0)$ & $19 / 21(90.5)$ & $33 / 41(80.5)$ & 0.000249 \\
& $R P\left(\beta_{2}\right)$ & $14 / 20(70.0)$ & $19 / 21(90.5)$ & $33 / 41(80.5)$ & 0.000167 \\
Spectral & $R P(\gamma)$ & $12 / 20(60.0)$ & $14 / 21(66.7)$ & $26 / 41(63.4)$ & 0.038629 \\
ratios & $r_{1}$ & $14 / 20(70.0)$ & $16 / 21(76.2)$ & $30 / 41(73.2)$ & 0.005298 \\
& $r_{2}$ & $15 / 20(75.0)$ & $19 / 21(90.5)$ & $34 / 41(82.9)$ & 0.000011 \\
& $r_{3}$ & $15 / 20(75.0)$ & $18 / 21(85.7)$ & $33 / 41(80.5)$ & 0.000015 \\
& $r_{4}$ & $15 / 20(75.0)$ & $17 / 21(81.0)$ & $32 / 41(78.0)$ & 0.000014 \\
\hline
\end{tabular}




\section{Table 4}

\begin{tabular}{|c|c|c|c|}
\hline Study & Data set & Parameters & Results \\
\hline Bennys et al. [23] & $\begin{array}{l}35 \text { AD patients and } \\
25 \text { elderly controls }\end{array}$ & $\begin{array}{c}\frac{R P(\theta)}{R P(\alpha)+R P\left(\beta_{1}\right)} \\
\frac{R P(\delta)+R P(\theta)}{R P(\alpha)+R P(\beta)}\end{array}$ & $\begin{array}{l}\text { Significant differences were found using the spectral ratios. } \\
\text { The classification results, applying a discriminant analysis } \\
\text { without a leave-one-out cross-validation scheme, showed a } \\
\text { sensitivity of } 65.7 \% \text { and a specificity of } 94.2 \% \text { at parieto- } \\
\text { temporo-occipital derivations, using the } R P(\theta) /\left[R P(\alpha)+R P\left(\beta_{1}\right)\right] \\
\text { ratio. Sensitivity of } 71.4 \% \text { and specificity of } 88.5 \% \text { were } \\
\text { obtained using the }[R P(\delta)+R P(\theta)] /[R P(\alpha)+R P(\beta)] \text { ratio. }\end{array}$ \\
\hline Jelic et al. [22] & $\begin{array}{l}14 \text { AD patients, } 12 \\
\text { mild cognitive } \\
\text { impairment (MCl) } \\
\text { patients and } 14 \\
\text { elderly controls }\end{array}$ & $\begin{array}{l}\frac{R P(\alpha)}{R P(\delta)} \\
\frac{R P(\alpha)}{R P(\theta)}\end{array}$ & $\begin{array}{l}\text { A strong correlation between cerebrospinal fluid (CSF) tau } \\
\text { levels and the spectral ratios was observed in } A D \text { patients, } \\
\text { whereas significant correlations between EEG slowing and } \\
\text { CSF tau levels were not found neither in } \mathrm{MCl} \text { patients nor in } \\
\text { healthy controls. }\end{array}$ \\
\hline Leuchter et al. [21] ${ }^{1}$ & $\begin{array}{l}49 \text { AD patients, } 29 \\
\text { multi-infarct } \\
\text { dementia (MDI) } \\
\text { patients and } 38 \\
\text { elderly controls }\end{array}$ & $\begin{array}{c}\frac{R P\left(\theta_{2}\right)+R P\left(\alpha_{1}\right)}{R P\left(\delta_{2}\right)+R P\left(\theta_{1}\right)} \\
\frac{R P\left(\alpha_{2}\right)}{R P\left(\delta_{2}\right)+R P\left(\theta_{1}\right)} \\
\frac{R P\left(\beta_{1}\right)}{R P\left(\delta_{2}\right)+R P\left(\theta_{1}\right)} \\
\frac{R P\left(\beta_{2}\right)}{R P\left(\delta_{2}\right)+R P\left(\theta_{1}\right)} \\
\frac{R P\left(\alpha_{2}\right)}{R P\left(\theta_{2}\right)+R P\left(\alpha_{1}\right)} \\
\frac{R P\left(\beta_{1}\right)}{R P\left(\theta_{2}\right)+R P\left(\alpha_{1}\right)} \\
\frac{R P\left(\beta_{2}\right)}{R P\left(\theta_{2}\right)+R P\left(\alpha_{1}\right)}\end{array}$ & $\begin{array}{l}\text { The parameters showed regional differences in distinguishing } \\
\text { between } A D \text { and MID patients and in their association with } \\
\text { age and cognitive status. The highest classification rates } \\
\text { were obtained using parameters based on low frequency } \\
\text { bands (i.e. } \delta \text { and } \theta \text { bands). In addition, the predicted } \\
\text { probabilities of true diagnosis enhanced substantially with the } \\
\text { increase in the number of parameters per subject used to } \\
\text { classify. }\end{array}$ \\
\hline Matousek et al. [24] & $77 \mathrm{AD}$ patients & $\frac{R P(\theta)}{R P(\alpha)}$ & $\begin{array}{l}\text { The spectral ratio was more correlated to the degree of } \\
\text { dementia than individual power measures. Given the fact that } \\
\text { the EEG slowing was highly dependent on the type of } \\
\text { regional syndrome affecting each patient, it was concluded } \\
\text { that EEG analysis would be more worthwhile in the early- } \\
\text { onset type of AD. }\end{array}$ \\
\hline Soininen et al. [20] & $\begin{array}{l}24 \mathrm{AD} \text { patients and } \\
14 \text { elderly controls }\end{array}$ & $\begin{array}{c}\frac{R P(\alpha)}{R P(\theta)} \\
\frac{R P(\alpha)}{R P(\delta)} \\
\frac{R P(\alpha)}{R P(\delta)+R P(\theta)} \\
\frac{R P(\alpha)+R P(\beta)}{R P(\delta)+R P(\theta)}\end{array}$ & $\begin{array}{l}\text { No significant differences between groups were found in } \beta \\
\text { power at the T6-O2 derivation. However, a significant } \\
\text { increase in the power of } \delta \text { and } \theta \text { bands and a decrease of } \alpha \\
\text { power was reported. The use of spectral ratios improved the } \\
\text { statistical results. Although all quantitative variables described } \\
\text { the EEG slowing, it was different at the time of AD diagnosis } \\
\text { and } 1 \text { year later. }\end{array}$ \\
\hline
\end{tabular}

\footnotetext{
${ }^{1}$ A particular subdivision of the conventional frequency bands was made in [21]: $\delta_{2}(2-4 \mathrm{~Hz}), \theta_{1}(4-6$ $\mathrm{Hz}), \theta_{2}(6-8 \mathrm{~Hz}), \alpha_{1}(8-10 \mathrm{~Hz}), \alpha_{2}(10-14 \mathrm{~Hz}), \beta_{1}(14-18 \mathrm{~Hz})$ and $\beta_{2}(18-22 \mathrm{~Hz})$.
} 